\title{
Lipedematous alopecia: clinical and histological analysis of the first male Chinese
}

Wei Wang ${ }^{1,2}$, Guizhi Zhou ${ }^{3}$, Yanfang Zhang ${ }^{3}$, Changping Yu³ and Furen Zhang ${ }^{1,3^{*}}$

\begin{abstract}
Background: Lipedematous alopecia $(\mathrm{LA})$ is a rare disorder clinically characterized by a thick boggy scalp and varying severity of hair loss, which primarily occurs in black female adults. In this study, we reported one male adult Chinese diagnosed with lipedematous alopecia for the first time.

Case presentation: A Chinese male, aged 20 years old, admitted to Shandong Provincial Hospital for Skin Diseases was diagnosed with LA. Clinical, histological and imaging data were collected and analyzed. Literature review was performed.

Results: Skin biopsy and pathological examination revealed the signs of increased subcutaneous adipose tissue, slight perivascular mononuclear infiltration into superficial dermis, adipocyte disruption and mucin deposition. CT scan demonstrated diffuse thickening of subcutaneous tissue in the occipital region. The symptoms were not significantly alleviated after 6-month combined therapy of prednisone and acupuncture.

Conclusions: We reported one male Chinese of LA, who was the first case reported in China. Clinical and pathological findings of this case deepened the understanding of $L A$.
\end{abstract}

Keywords: Lipedematous alopecia, Male, Histological examination, Skin biopsy

\section{Background}

Lipedematous scalp is a rare condition manifested as an increased thickness of subcutaneous tissue in the scalp gives rise to a soft spongy appearance of the surface and occasionally causes pruritus and pain in the affected area. When hair loss is also associated with the condition, it is described as lipedematous alopecia (LA). LA is a rare condition characterized by a thick, boggy scalp with varying degrees of hair loss that occurs in adult black females, with no clearly associated medical or physiological conditions. However, the underlying pathogenesis remains elusive (Martin et al. 2005; Ko et al. 2011; Fair et al. 2000). Pathologic finding reveals an approximately doubled scalp thickness resulting from expansion of the

\footnotetext{
*Correspondence: furenlucky@sina.com

${ }^{3}$ Shandong Provincial Institute of Dermatology and Venereology, Shandong Academy of Medical Sciences, No. 27397 Jingshi Road, Jinan 250022, Shandong Province, People's Republic of China Full list of author information is available at the end of the article
}

subcutaneous fat layer in the absence of adipose tissue hypertrophy or hyperplasia. Interestingly, LA is primarily encountered in the female. In this study, we firstly reported one male Chinese patient diagnosed with LA. Clinical, pathological and imaging features were retrospectively analyzed, adding clinical evidence to understand of this rare clinical entity.

\section{Case presentation}

A 20-year-old Chinese man presented with a 4-year history of scalp pruritus and hair loss. At the age of 16, he started to suffer from a gradual loss of hair, initially from the vertex and occipital areas, but eventually extending to the entire scalp during subsequent 1 month after the onset of symptoms. He reported no family history of a similar entity or trauma. Physical examination revealed alopecia of the entire scalp and the remaining hair was short and less than $2 \mathrm{~cm}$, as shown in Fig. 1a. Upon palpation, the scalp felt mildly tender and had a boggy 

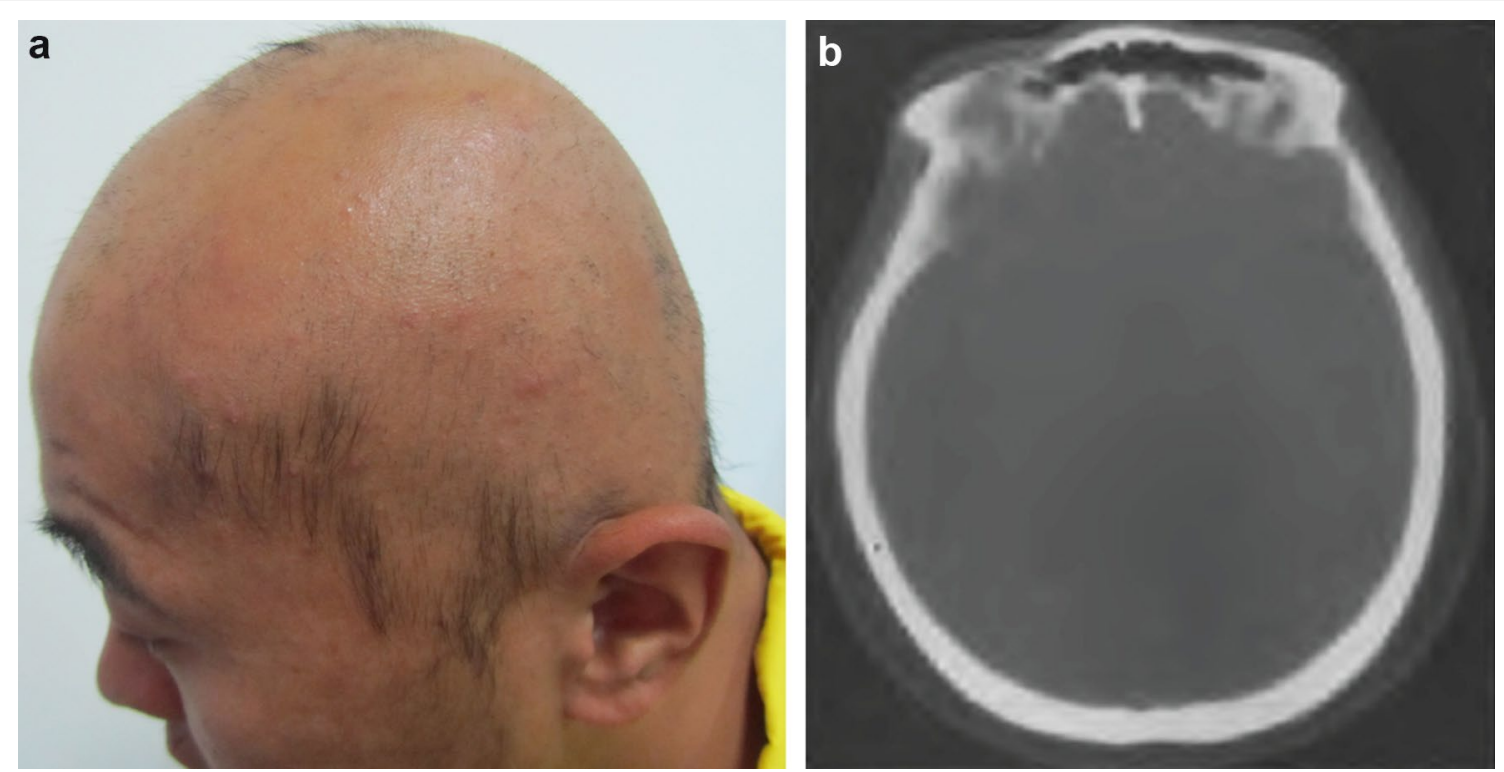

Fig. 1 a Clinical manifestation showing alopecia accompanied by thickened scalp. b CT scan showing thickening scalp with marked expansion of subcutaneous adipose tissue approximately $15 \mathrm{~mm}$ in the occipital region

spongy consistency. The scalp could be easily pressed down to the underneath bone, but immediately restored to original shape. Computer tomography $(\mathrm{CT})$ scan demonstrated diffuse thickening of the subcutaneous tissues in the occipital region, which was measured as approximately 15 mm (Fig. 1b).

Chemical panel, complete blood count, thyroid function test, and antinuclear antibody titers yielded normal outcomes. Trichoscopy revealed no yellow dots, black dots, or exclamation mark hair. Hair pull test was negative. No hair loss was observed in other body parts. Skin biopsy showed increased subcutaneous adipose tissue and mild perivascular mononuclear infiltration into the superficial dermis (Fig. 2a). Scant perifollicular mononuclear infiltration accompanied by perifollicular fibrosis was equally observed in Fig. 2b. The count of hair follicle was decreased. Adipocyte disruption and mucin deposition were also demonstrated, as illustrated in Fig. 2c, d.

According to the findings and outcomes, he was eventually diagnosed with LA. After the diagnosis was validated, the patient was administered with prednisone at a daily dosage of $30 \mathrm{mg}$ in combination with acupuncture therapy for consecutive 6 months. Acupuncture therapy has been reported to accelerate the blood circulation and stimulate the acupoint. However, the symptoms of hair loss and the thickening of subcutaneous fat tissue were not significantly mitigated after this combined therapy.

\section{Discussion}

LA is an extremely rare condition firstly described by Coskey in 1961, dominantly occurring in black female adults (Kavak et al. 2008; Blaheta et al. 1999). To date, more than 80 LA cases have been reported in the literatures (Müller et al. 2012), a majority of whom are black female patients including merely three Asians. Moreover, LA is primarily encountered in female patients, and only four male cases have been reported yet. Common symptoms of LA include joint and skin hyperelasticity, discoid lupus, diabetes mellitus and acute kidney failure (Ko et al. 2011). LA is pathologically characterized by increased subcutaneous adipose tissues (Yasar et al. 2011). The scalp thickness of LA varies from 10 to $16 \mathrm{~mm}$, significantly thicker compared with $5.5 \mathrm{~mm}$ of healthy counterparts (Yasar et al. 2007).

In present investigation, we reported the first male patient diagnosed with LA in China, suggesting that the incidence of LA might be more geographically and ethnically widespread than previously thought (El Darouti et al. 2007). Moreover, the incidence of LA probably does not significantly differ between both genders. Histopathological findings revealed adipocyte disruption besides increased subcutaneous adipose tissue in this male patient, which has been seldom reported. We assumed that the incidence of LA may be related to adipocyte disruption, which warrants further investigation. Scant perifollicular mononuclear infiltration and perifollicular fibrosis are inconsistent pathological features of LA patients. To date, only two cases have been reported 

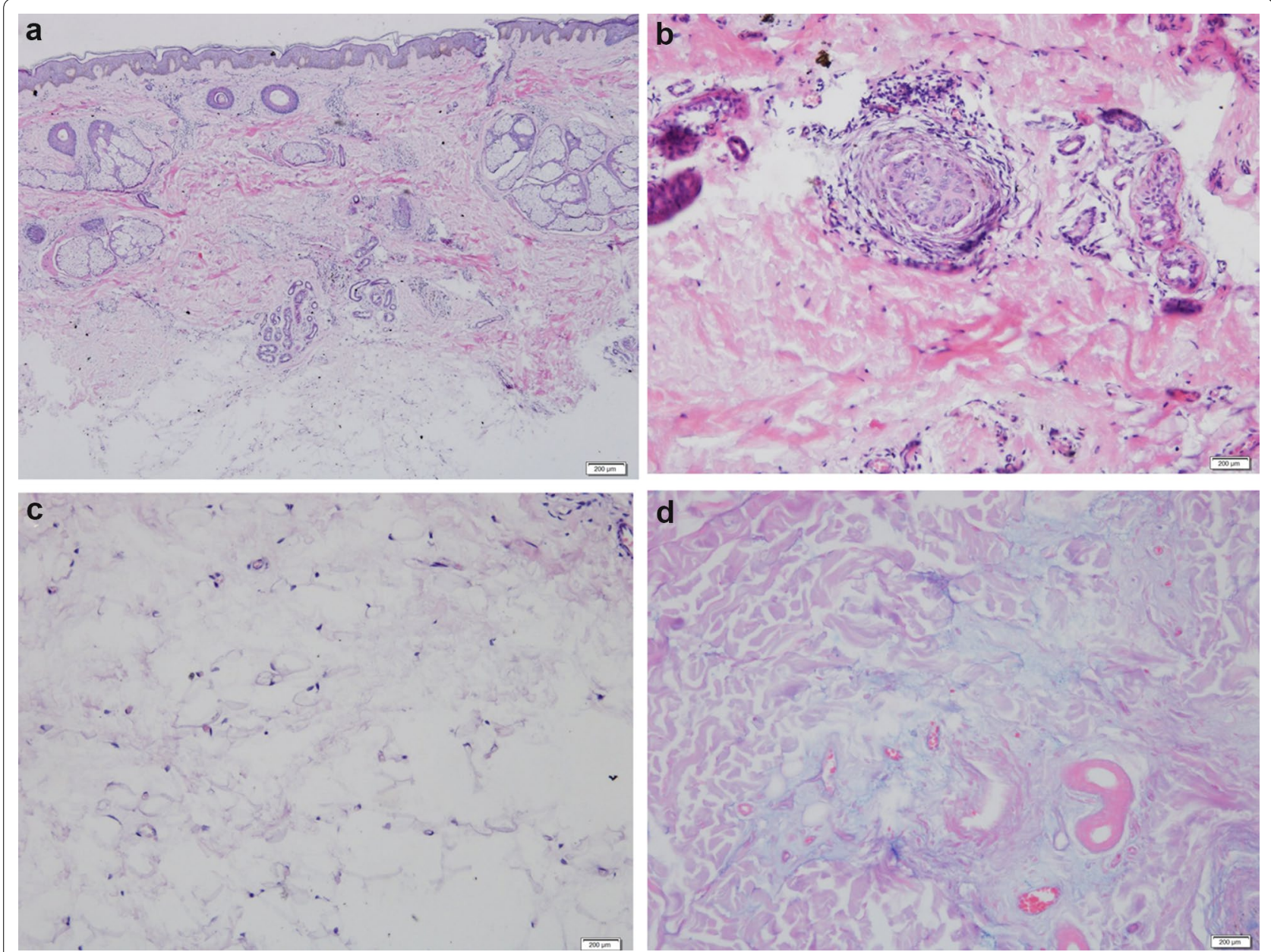

Fig. 2 a H\&E staining revealing mild perivascular mononuclear infiltration into the superficial dermis and increased subcutaneous adipose tissue (×100 magnification). b H\&E staining showing perifollicular fibrosis (×400 magnification). c H\&E staining revealing disruption of adipocytes ( 4000 magnification). d Alcian blue staining showing mucin deposition in the dermis ( $\times 400$ magnification)

to have mucin deposition in the dermis or subcutis. The patient in present study presented with mucin in the dermis and subcutis. The causes and clinical significant of this sign remain to be further elucidated.

In our case, multiple symptoms including hair loss, thick and boggy scalp were found simultaneously and the entire scalp rapidly evolved with only 1 month, indicating that LS and LA are separate entities. Mounting evidence supported an autoimmune origin of LA, although the exact cause and triggering factors are still unknown. To validate the diagnosis of LA, clinical, histopathological and other findings should be performed simultaneously. Currently, multiple surgical (Yip et al. 2008) and medication therapies have been applied to alleviate the symptoms of LA and LS, whereas poor clinical efficacy has been achieved. Yip et al. successfully treat a 67 -year-old woman with LA by using surgical debulking combined with scalp reduction and acceptable efficacy was obtained after 12-month follow-up (Cabrera et al. 2015). In a recent investigation, Cabrera et al. (2015) reported that a female patient diagnosed with LA was properly healed after administration of mycophenolate mofetil. Optical treatment of LA remains to be explored by large sampling size investigations.

\section{Abbreviations \\ LA: lipedematous alopecia; CT: computer tomography.}

\section{Authors' contributions}

All authors have made substantial contributions to conception and design, or acquisition of data, or analysis and interpretation of data; WW, GZZ, YFZ, CPY and FRZ have been involved in drafting the manuscript or revising it critically for important intellectual content; all authors have given final approval of the version to be published; and all authors agree to be accountable for all aspects of the work in ensuring that questions related to the accuracy or integrity of any part of the work are appropriately investigated and resolved. All authors read and approved the final manuscript. 


\section{Author details}

${ }^{1}$ Shandong Provincial Hospital for Skin Diseases, Shandong University, Jinan 250022, Shandong Province, People's Republic of China. ${ }^{2}$ Department of Dermatology, Jining No. 1 People's Hospital, Jining, Shandong Province, People's Republic of China. ${ }^{3}$ Shandong Provincial Institute of Dermatology and Venereology, Shandong Academy of Medical Sciences, No. 27397 Jingshi Road, Jinan 250022, Shandong Province, People's Republic of China.

\section{Competing interests}

The authors declare that they have no competing interests.

\section{Informed consent}

Written informed consent was obtained from the patient for publication of this Case report and any accompanying images

Received: 1 June 2016 Accepted: 29 September 2016

Published online: 10 October 2016

\section{References}

Blaheta RA, Leckel K, Wittig B, Zenker D, Oppermann E, Harder S, Scholz M, Weber S, Encke A, Markus BH (1999) Mycophenolate mofetil impairs transendothelial migration of allogeneic CD4 and CD8 T-cells. Transplant Proc 31:1250-1252
Cabrera R, Larrondo J, Whittle C, Castro A, Gosch M (2015) Successful treatment of lipedematous alopecia using mycophenolate mofetil. Acta Derm Venereol 95(8):1011-1012

El Darouti MA, Marzouk SA, Mashaly HM, El Nabarawi EA, Abdel-Halim MR, El Komy MM, Hafez VG, Abdel Reheem HM, Moussa KM (2007) Lipedema and lipedematous alopecia: report of 10 new cases. Eur J Dermatol 17:351-352

Fair KP, Knoell KA, Patterson JW, Rudd RJ, Greer KE (2000) Lipedematous alopecia: a clinicopathologic, histologic and ultrastructural study. J Cutan Pathol 27(1):49-53

Kavak A, Yuceer D, Yildirim U, Baykal C, Sarisoy HT (2008) Lipedematous scalp: a rare entity. J Dermatol 35(2):102-105

Ko JH, Shih YC, Toh CH, Lee HE, Kuo TT, Hui RC (2011) Lipedematous alopecia with mucinosis: report of the first case in Taiwan. Dermatol Sin 29:25-28

Martin JM, Monteagudo C, Guijarro J, Liombart B (2005) Lipedematous scalp and lipedematous alopecia: a clinical and histologic analysis of 3 cases. J Am Acad Dermatol 52:152-156

Müller CS, Niclou M, Vogt T, Pföhler C (2012) Lipedematous diseases of the scalp are not separate entities but part of a spectrum of lipomatous lesions. J Dtsch Dermatol Ges 10:501-507

Yasar S, Mansur AT, Goktay F, Sungurlu F, Aker FV, Ozkara S (2007) Lipedematous scalp and lipedematous alopecia: report of three cases in white adults. J Dermatol 34:124-130

Yasar S, Gunes P, Serdar ZA, Tosun I (2011) Clinical and pathological features of 31 cases of lipedematous scalp and lipedematous alopecia. Eur J Dermatol 21:520-528

Yip L, Mason G, Pohl M, Sinclair R (2008) Successful surgical management of lipoedematous alopecia. Australas J Dermatol 49:52-54

\section{Submit your manuscript to a SpringerOpen ${ }^{\circ}$ journal and benefit from:}

- Convenient online submission

- Rigorous peer review

- Immediate publication on acceptance

- Open access: articles freely available online

- High visibility within the field

- Retaining the copyright to your article 